of glands are also changed. In other words, two manifestations of an entirely dissimilar character and which we are accustomed to study separately are here found to be coincident and, beyond any doubt, dependent upon a common origin.

The lack of time and opportunity have prevented my making a study of this question. There is no doubt, whatever, in my mind that other causes of a like nature have occurred, but the importance of the graver malady as well as its comparative rarity completely overshadowed the importance of the symptom which probably is one of frequent occurrence-the bromidrosis.

A feature which was observed and which might partially account for the fotid sweat is the fact noted in the history that there was no pigmentation of the mucous membrate of the mouth. The explanation that this would furnish would be that the energy usually directed to the mucous membrane had been diverted to other channels, or, in other words, the perverted innervation was transferred from the mucous membrane to its external congener, the skin. This process is not an unusual one. We observe it, for instance, in erythema nodosum in which the eruption rapidly disappears to give way to a bronchitis, and this in turn leaves to be followed by a recurrence of the eruption.

In this short clinical contribution I have merely desired to sketch an interesting condition, and, so far as I know, a peculiar case. While the literature of Addison's disease is plentiful there is really but little that is tangible. In the light of modern anatomical investigations, however, we can see the promise of great improvement in the future therapeutical measures to be' employed, as well as in the more thorough and intelligent pathological investigations which will be made.

5 South Broadway.

NOTES ON A CASE OF TETANY.

Read in the Section of Diseases of Children at the Forty-first Annual Meeting of the American Medical Association,

BY JACOB SCHNECK, M.D., OF MT. CARMEL, ILL.

Under the various names of tetany, tetanella, idiopathic muscular spasm, and carpo-pedal spasm, we find described a variety of cases which have, as prominent symptoms, more or less tonic contractions of the muscles of various parts of the body, beginning usually at the extremities and passing, in somewhat regular succession, to the muscles of the body and head. The consciousness of the individual is not disturbed during the paroxysm.

The symptoms of this disease trench closely on those of a number of the functional neuroses; and it is probable that many of the cases reported un- der this heading are really spasms due to reflex irritation, caused by teething or a disordered condition of the alimentary canal; or to irritation produced in the nerve-centres by the distal ends of nerves, leading to them, being involved in cicatrices or other inflammatory products ; these cases are permanently cured by removing the cause of the irritation. It is also probable that in this category cases have been reported which should be described under the title of hysteria.

The causes usually assigned as producing tetany are exceedingly numerous and various; chronic and debilitating diarrhœea, lactation and pregnancy, stand first, perhaps. Typhoid fever, rheumatism, measles, pneumonia, Bright's disease, small-pox, cholera, malaria, exposure to cold, anæmia, sexual excesses, alcoholism, excision of thyroid gland, intestinal worms, disordered stomach from undigested food, lead poisoning, and epidemic influences, have all been thought to be causes. In reading over the reports of some cases one is led to believe that many of the above named causes are only coincidences, and had nothing to do with inducing the disease. An important diagnostic point of this disease is the tendon-reflex action produced by pressing on the large vessels and nerves (Trousseau's symptoms). This is not always present, but in no other disease has it ever been recorded.

Chvostek was the first to direct attention to the increased mechanical excitability of the muscles and nerves in this disease; he found in many cases that during the latent period the calf-muscles were slightly contracted. During the paroxysms consciousness is intact; and the muscuar spasms are tonic.

The present light on the pathology of this disease points to the nerve-centres as the site of disturbance.

Herz thought the disease was due to anæmia of the spinal cord. A. Jacobi attributes it to meningeal hyperæmia. Gowers teaches that it is probable that the primary pathological lesion. is in the motor cells of the cerebral and spinal structures; and that thus is explained the tendon-reflex symptoms usually present in this disease. Whatever the pathology may be, there appears to be an inability to inhibit in the nervous and muscular organs.

I am induced to offer these notes on the following case for three principal reasons:

I. Tetany is a disease whose limits and symptomatology are not well defined.

2. The case which fell into my hands had the signs, usually given as belonging to this disease, well-marked. In addition there were other prominent symptoms, which I do not find given in any of the accounts of the disease which I have consulted, viz.: the absence of normal reflex excitability in the extensor muscles, as shown by the want of the tendon reflex action of the exten- 
sors of both the upper and lower extremities. And also the Argyll-Robertson pupil; both of which signs improved and became normal pari passu with the diminution of the number and severity of the attacks.

3. For the purpose of putting on record the treatment and its result, so that the profession may give it a further trial.

Case.-Oct. 23, 1889, Clara I. Brine, of Grayville, Ill., was brought to my office; she being a well developed, bright and healthy looking girl of ten years. The mother and father, who accompanied her, were both above the average in size, of intelligent bearing, and barring a few slight attacks of unimportant sickness, had always been healthy. Their five other children were all well developed. Clara had been healthy until three years since, when she suffered from some ailment of the bladder, but which was of temporary duration. Near two years ago, while in her usual health, she was suddenly seized with a swimming sensation and fell backwards on the floor; not, however, losing consciousness. Attacks of this character occurred at frequent intervals for nearly one year. She usually avoided falling by holding to some object until the sensation passed off. The physician who treated her told the parents the attacks were caused by some heart disease. Gradually these paroxysms were ushered in, or preceded, by more or less tonic cramps of the lower limbs; felt most severely at the knees; when the feet were involved, the toes would be drawn together and towards the sole of the foot. Later the upper extremities, and finally the jaws, became involved in the tonic contractions; but as they.did so, the dizziness became less and the cramps of the lower limbs grew lighter, and for the last six months had ceased entirely; while those of the upper extremities, neck, and jaws, had grown correspondingly more severe and more frequent; averaging about ten a day; being most frequent during the forenoon, but never occurring during sleep. In some of the most severe attacks there was decided opisthotonos. In all those attacks consciousness was never lost. During the past year she had been treated by a physician for epilepsy. During the time of her visit to my office, while I was familiarizing myself with a history of her case, she was seized with a severe paroxysm, while in the midst of a sentence, and when not in the least excited. It came without any premonition and involved both upper extremities, and the muscles of the neck and jaws, in the order named. The muscles were so rigid that she was unable to speak, swallow, or move the head; the elbowjoints were flexed and the arms held rigidly from the side; the right hand was closed into a hard fist: the fingers of the left were first irregularly over-extended, then drawn together, the two outer coming under the two inner ones, while the thumb was drawn down to the pailm, forming the obstetric hand. The face became full and flushed. This condition lasted near two minutes. The paroxysm was accompanied by radiating pains in the parts involved. "A sharp pain, with other pains shooting out from it," was her expression. She complained, after the attack, of numbness and formication in the muscles affected, also of ringing in the ears. Examination revealed slight cedema of the hands and face; tendon-reflex of knees and wrist entirely absent; Argyll-Robertson pupil well marked; pulse i 20 per minute, small and hard. Had always suffered slightly from constipation; appetite good; sleeps well and quietly; temperature normal. There was no tenderness along the spine or over the ovaries, or any other part of the body; not subject to excessive perspiration ; nervo-muscular irritability could not be produced by raking finger-nail in front of ear; there was no nausea or vomiting after attacks; she had not been a bottlefed infant; had never suffered from rickets or iaryngismus; did not pass large quantities of urine after paroxysms; pressure on the large blood-vessels or nerves did not bring on an attack; applying cold water to hands failed to cut short a paroxysm.

The treatment and progress of the case were as follows :

October 23, 1889 .

R. Liquor potass. arsenitis, $3 \mathrm{j}$

Sodæ brom., $\breve{z}$ ss.

Aqua, ad. 3 iv. $m$.

Sig.-One teaspoonful after meals.

R. Antifebrin, gr. xxx.

Divide into 15 capsules.

Sig.-One capsule before meals.

October 3 I, symptoms very slightly improved; averaged five paroxysms per day since the former visit; pulse I 2O; tendon-reflex of the extremities imperceptable. Argyll-Robertson pupil still marked.

Thinking she might be afflicted with worms, I gave the following:

R. Hydrarg. chlor, mitis, gr. ij.

Sodæ bicarb. santonine, āā gr. vj. Mix fiat chart No. vj.

Sig.-One powder at 4,6 and 8 P.M. on consecutive nights.

Then to begin the following:

R. F1. ext. conium, $3 \mathrm{j}$.

Sig.-Eight drops after each meal.

R. Glonoin, 3 iv.

Sig. - Two drops two hours after meals.

Nov. I 2.- - Had passed no worms from santonine. During the first six days she had nineteen paroxysms, and none during the last five. Continues the two last prescriptions.

Dec. 4.-Had no paroxysm since last visit; tendon reflex of both upper and lower extremities nearly normal; pupil responds properly to light. Continued the nitro-glycerine and stopped the conium. 
Feb. 3, I89o.-Had no attack since last report; continued treatment.

May 10, 1890.- Reports herself as entirely well, and has taken no medicine for two months.

\section{DIAGNOSIS AND OPERATIVE TREAT- MENT OF GUNSHOT WOUNDS OF THE STOMACH AND INTESTINES.}

Read by Invitation in the Surgical Section of the Tenth International Medical Congress, August 8, 1890 .

BY N. SENN, M.D., PH.D.,

OF MILWAUKEE, wis.

ATTENDING SURGEON MILWAUKEE HOSPITAL; PROFESSOR PRINCIPLES OF SURGIERY AND SURGICAL PATHOLOGY IN RUSH MEDICAL COLLEGE, CHICAGO, ILI.

(Concluded from p. 363 .)

2. Treatment of Gunshot Wounds of the Stomach and Intestines.

The propriety of surgical interference in cases of gunshot wounds of the stomach and intestines will depend on one of two things :

I. Dangerous internal hæmorrhage.

2. Visceral wounds of the stomach or intestines large enough to permit of extravasation and the escape of hydrogen gas on applying the diagnostic test.

Alarming intraperitoneal hæmorrhage furnishes an urgent indication for treatment by laparotomy irrespective of the existence of the visceral lesions of the gastro-intestinal canal. In these days of aggressive surgery it would indeed be unjustifiable to permit a patient to die slowly but surely from bæmorrhage from an intra-abdominal wound which in itself is amenable to successful treatment by prompt surgical means.

The signs and symptoms of profuse internal hæmorrhage come on soon after the injury has been inflicted, and are so plain and significant that they can hardly be misinterpreted even upon a brief and superficial examination. The progressive acute anæmia, the conditions of the pupils and pulse, and the complexus of nervous symptoms which attend alarming internal hæmorrhage present a clinical picture which, when once seen, is never forgotten and always recognized.

In such cases no time should be lost in looking for evidences of the existence of wounds of the gastro-intestinal canal, or in applying the hydrogen gas test, as the prime and most urgent indication is to gain access to the bleeding vessels by opening the abdomen in the median line, and then to arrest the hæmorrbage at once by compression until the bleeding points can be secured by ligation or the antiseptic tamponnade. After the hæmorrhage has been arrested, the whole gastrointestinal canal, or such part of it as, from the course of the bullet, is known to have been exposed to injury, is tested by insufflation of hydrogen gas for the purpose of ascertaining in as short a time as possible, the existence, location and number of perforations.

In cases in which, on account of the absence of dangerous hæmorrhage, the symptoms are less urgent, it is important to determine the necessity of treatment by laparotomy by resorting to the hydrogen gas test for the purpose of diagnosticating the existence and size of perforations of the gastro-intestinal canal. If through insufflation can be made, it is proof positive that no perforations exist, or, if present, that they are too small for extravasation to occur, and in the absence of other indications for abdominal section it is advisable to pursue a conservative course of treatment. Such patients are given an opiate to diminish the peristaltic action of the bowels; an absolute diet is ordered and rest in bed enforced for at least a week, while infection from without is prevented by subjecting the external wound to rigid antiseptic treatment.

If the hydrogen gas test, either by rectal insufflation or direct inflation of the stomach, yields a positive result, sufficient and reliable information has been gained to warrant laparotomy, as the surgeon has then satisfied himself that he has to deal with an injury which, if not properly treated, is almost certain to result in the death of the patient.

\section{Laparotomy for Gunshot Wounds of the Stomach and Intestines.}

I have already insisted that in every perforating gunshot wound of the abdomen, all necessary preparations for laparotomy should be made before the existence of visceral wounds of the stomach or intestines has been demonstrated by the application of the hydrogen gas test, as the latter procedure necessitates the administration of an anæsthetic. The stomach can be inflated without an anæsthetic and the existence or absence of perforations demonstrated before any definite plan of treatment is proposed, but rectal insufflation of hydrogen gas should never be undertaken in the diagnosis of gunshot wounds of the gastrointestinal canal without complete anæsthesia.

Before I was able to demonstrate the existence of visceral wounds of the stomach and intestines without opening the abdominal cavity, I never succeeded in obtaining the consent of the patient and friends to make a laparotomy in cases of gunshot wounds of the abdomen; but since I have been able to tell the people that the course to be pursued will depend upon the result of the test, I have not met with a single refusal. The hydrogen gas flame at the wound of entrance is an argument more potent than words in convincing laymen of the necessity of active surgical interference. If the public understands that the abdomen will only be opened after the surgeon has satisfied himself and has demonstrated the existence of a mortal injury, the patient and his friends will be only too anxious to avail them- 